\title{
New Silybin Scaffold for Chemical Diversification: Synthesis of Novel 23-Phosphodiester Silybin Conjugates
}

\author{
Armando Zarrelli, Valeria Romanucci, Marina Della Greca, Lorenzo De Napoli, Lucio Previtera, Giovanni Di Fabio* \\ Department of Chemical Sciences, University of Napoli 'Federico II', via Cintia 4, 80126 Napoli, Italy \\ Fax +39(081)674393; E-mail: difabio@unina.it
}

Received: 01.10.2012; Accepted after revision: 02.11.2012

\begin{abstract}
Silybin is the major component (ca. 30\%) of the silymarin complex extracted from the seeds of Silybum marianum, with multiple biological activities operating at various cell levels. As an ongoing effort toward the exploitation of natural products as scaffolds for chemical diversification at readily accessible positions, we present here an efficient synthetic procedure to obtain new 23-phosphodiester silybin conjugates with different labels. A key point in our approach is the new 3,5,7,20-tetra- $O$-acetylsilybin-23-phosphoramidite, useful for a variety of derivatizations following a reliable and well-known chemistry. The feasibility of the procedure has been demonstrated by preparing new 23 -silybin conjugates, exploiting standard phosphoramidite chemistry.
\end{abstract}

Key words: natural products, flavonolignans, silybin, phosphorylation, drugs

Flavonoids and flavonolignans are widely distributed among various citrus plants and are frequently found in the human diet. They may act as enzyme inhibitors, freeradical scavengers, antitumor agents, antibacterial agents, anti-inflammatory agents, and antioxidants. ${ }^{1-3}$

Silybin (Figure 1) is the major component (ca. 30\%) of the silymarin complex extracted from the seeds of Silybum marianum consisting of two diastereomers $\mathbf{A}$ and $\mathbf{B}$ in a ratio of approximately $1: 1$.

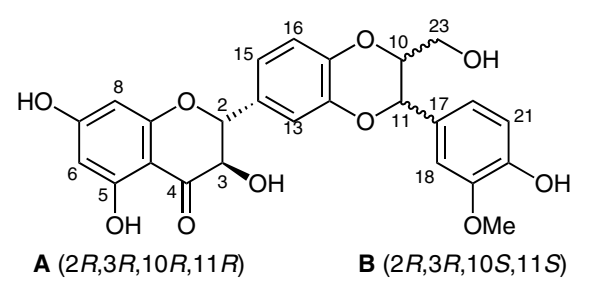

Figure 1 Chemical structure of silybin $\mathbf{A}$ and silybin B

Silybin is a natural compound with multiple biological activities operating at various cell levels most of them related to its radical-scavenging activity. Along with the beneficial activities resulting from the antioxidant and radical-scavenging properties, ${ }^{3,4}$ silybin has recently received attention due to its anticancer and chemopreventive actions, ${ }^{5,6}$ as well as hypocholesterolemic, cardioprotective, and neuroprotective activities. ${ }^{3,7}$ In vivo applications of silybin are rather hampered by its very low

SYNLETT 2013, 24, 0045-0048

Advanced online publication: 04.12.2012

DOI: 10.1055/s-0032-1317688; Art ID: ST-2012-D0837-L

(C) Georg Thieme Verlag Stuttgart · New York bioavailability. In an attempt to improve its biological properties and facilitate in vivo applications of silybin, only limited structural modifications have been proposed $^{3,8-12}$ and the available analogues are still unsatisfactory. Therefore new synthetic approaches for selectively modifying silybin are of interest.

As a part of our continuing research effort towards the synthesis of new natural product analogues,,${ }^{13,14}$ we present here the preliminary results of a efficient synthetic procedure to obtain new 23-phosphodiester silybin conjugates with different labels.

The introduction of a phosphate group may bring pharmaceutical and pharmacokinetic benefits. ${ }^{15}$ Conjugation is usually considered as an efficient route in drug discovery to improve the biological properties of a large number of drugs and can improve the bioavailability and delivery as well as the biological activity.

We chose to start from the new 23-phosphoramidite building block 3 (Scheme 1) which could be transformed into a series of conjugates using a solution-phase parallel array protocol, exploiting standard and reliable phosphoramidite chemistry. ${ }^{15}$ We initially converted silybin (1) into its 23-ODMT ether by a reaction with DMT-chloride in pyridine at $50{ }^{\circ} \mathrm{C}$. After exhaustive acetylation with an excess of acetic anhydride in pyridine, subsequent treatment with $5 \%$ formic acid in dichloromethane allowed the removal of the DMT protecting group to give $\mathbf{2}$ in $75 \%$ yield and this could be converted into the corresponding phosphoramidite derivatives 3 . Thus intermediate $\mathbf{2}$ was reacted with 2-cyanoethyl- $N, N$-diisopropylamino-chlorophosphoramidite and DIPEA in anhydrous dichloromethane. In these preliminary studies, the silybin used was a mixture of diastereomers, and the derivatives 3 were obtained as a mixture of inseparable diastereomers, although the ${ }^{1} \mathrm{H}$ NMR and ${ }^{31} \mathrm{P}$ NMR spectra appeared to be of a single compound. After purification the identities of compounds 3 , obtained in good yields (65\%), were confirmed by NMR $\left({ }^{1} \mathrm{H},{ }^{13} \mathrm{C}\right.$, and $\left.{ }^{31} \mathrm{P}\right)$ and ESI-HRMS analysis. ${ }^{16}$

Subsequently we selected a group of model molecules having a free hydroxyl group (A-E, Scheme 1), useful for coupling with the key intermediate $3 .{ }^{17}$ In particular, we selected molecules known for their ability to act as molecular carriers (steroids, bile acids), ${ }^{18-21}$ to improve water solubility (polyethers) 22 and as radical scavengers (nucleosides). ${ }^{23,24}$ While $\mathbf{A}, \mathbf{B}$, and $\mathbf{C}$ are commercially available, $\mathbf{D}$ and $\mathbf{E}$ were efficiently obtained starting from 2'deoxyadenosine and $3 \alpha, 7 \alpha, 12 \alpha, 24$-tetrahydroxycholane, 
<smiles>COc1cc(C2Oc3cc([C@@H]4Oc5cc(O)cc(O)c5C(=O)C4O)ccc3OC2CO)ccc1O</smiles><smiles>COc1cc(OC(C)=O)c2c(c1)O[C@@H](c1ccc3c(c1)OC(c1ccc(OC(C)=O)c(OC)c1)C(CO)O3)C(OC(C)=O)C2=O</smiles>

d)

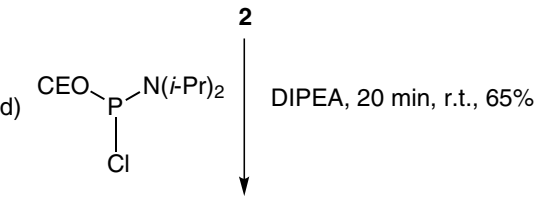

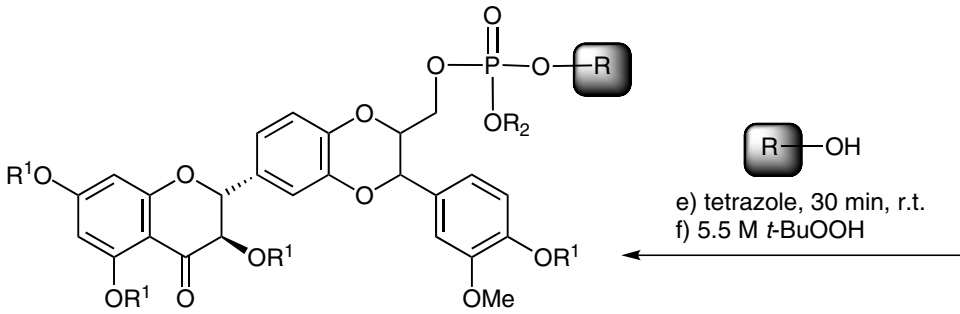<smiles>CCCCNP(OCC)OCC1Oc2ccc([C@@H]3Oc4cc(OC(C)=O)cc(OC(C)=O)c4C(=O)C3OC(C)=O)cc2OC1c1ccc(OC(C)=O)c(OC)c1</smiles>

$\mathrm{NH}_{4} \mathrm{OH}, \mathrm{MeOH}$ 4a-e $R^{1}=A c ; R^{2}=C E=2-c y a n o e t h y l$ $1 \mathrm{~h}, \mathrm{r.t}$.

5a-e $\mathrm{R}^{1}=\mathrm{R}^{2}=\mathrm{H}$

$\mathbf{5 a}=68 \% ; \mathbf{5 b}=55 \% ; \mathbf{5 c}=72 \% ; \mathbf{5 d}=70 \% ; \mathbf{5 e}=65 \%^{\star}$

Scheme 1 Synthesis of 23-phosphodiester silybin conjugates 5a-e. *Yields calculated from starting product 3; CE = cyanoethyl

respectively. Exploiting the different reactivity of hydroxyl groups our studies were carried out using TBDMSCl or DMTCl for the transient protection of the primary $\mathrm{OH}$ moiety while all other groups were protected by acetylation. 2'-Deoxyguanosine and $3 \alpha, 7 \alpha, 12 \alpha, 24$-tetrahydroxycholane were protected by reaction with TBDMSCl and DMTCl, respectively. ${ }^{25,26}$ The products were acetylated with acetic anhydride to yield fully protected products. Finally, the TBDMS group was cleaved by $\mathrm{Et}_{3} \mathrm{~N} \cdot 3 \mathrm{HF}$ and DCA to yield $\mathrm{N}^{2}$-acetyl-2', $3^{\prime}$ - $O$-diacetyl-deoxyguanosine (D) and $3 \alpha, 7 \alpha, 12 \alpha-O$-triacetyl-24-hydroxycholane (E) in overall yields of 80 and $75 \%$, respectively.

The coupling of $\mathbf{3}$ with $\mathbf{A}-\mathbf{E}$ (Scheme 1) was carried out by using the classic coupling reagent $(0.45 \mathrm{M}$ tetrazole in $\mathrm{MeCN}$ ), and then treatment with $5.5 \mathrm{M}$ tert-butyl hydroperoxide solution in decane led to phosphotriesters $\mathbf{4 a}-\mathbf{e}$. After purification by flash chromatography, the derivatives $\mathbf{4}$ were treated with concemtrated aqueous ammonia and $\mathrm{MeOH}(1: 1, \mathrm{v}: \mathrm{v})$ at room temperature, allowing full deprotection, leading to the desired phosphodiester derivatives 5a-e in good yields (Scheme 1) ${ }^{27}$ All final derivatives $\mathbf{5 a}-\mathbf{e}$ were purified by flash chromatography and then characterized by NMR $\left({ }^{1} \mathrm{H}\right.$ and $\left.{ }^{31} \mathrm{P}\right)$ and MS analysis. All the intermediates and final derivatives were obtained as mixtures of two diastereomers, that were inseparable by chromatography, although the NMR spectra of many of them appeared to indicate a single compound.

In conclusion a facile and efficient protocol for the synthesis of a broad array of new 23-phosphodiester silybin structured conjugates has been achieved. The feasibility of this procedure has been demonstrated by preparing new 23-silybin conjugates, exploiting standard phosphoramidite chemistry. A key point in our strategy is the new silybin building block $\mathbf{3}$, useful for a variety of derivatizations following established chemistry. In principle, this methodology can be readily extended to other molecules which have a free hydroxyl group. ${ }^{15,28}$

\section{Acknowledgment}

This study was supported by AIPRAS Onlus (Associazione Italiana per la Promozione delle Ricerche sull'Ambiente e la Saluta umana). We also thank Dr. Vincenzo Perino of the CIMCF, Università degli Studi di Napoli 'Federico II', for access to NMR facilities.

Supporting Information for this article is available online at http://www.thieme-connect.com/ejournals/toc/synlett.

\section{References and Notes}

(1) Havesteen, B. Pharmacol. Ther. 2002, 96, 67. 
(2) Ferrazzano, G. F.; Amato, I.; Ingenito, A.; Zarrelli, A.; Pinto, G.; Pollio, A. Molecules 2011, 16, 1486.

(3) Gažák, R.; Walterová, D.; Křen, V. Curr. Med. Chem. 2007, $14,315$.

(4) Lu, P.; Mamiya, T.; Lu, L. L.; Mouri, A.; Niwa, M.; Hiramatsu, M.; Zou, L. B.; Nagai, T.; Ikejima, T.; Nabeshima, T. J. Pharmacol. Exp. Ther. 2009, 331, 319.

(5) Hogan, F. S.; Krishnegowda, N. K.; Mikhailova, M.; Kahlenberg, M. S. J. Surg. Res. 2007, 143, 58.

(6) Sharma, G.; Singh, R. P.; Chan, D. C.; Agarwal, R. Anticancer Res. 2003, 23, 2649.

(7) Zhao, H.; Brandt, G. E.; Galam, L.; Matts, R. L.; Blagg, B. S. J. Bioorg. Med. Chem. Lett. 2011, 21, 2659 ; and references cited therein.

(8) Gažák, R.; Valentová, K.; Fuksová, K.; Marhol, P.; Kuzma, M.; Medina, M. A.; Oborná, I.; Ulrichová, J.; Křen, V. J. Med. Chem. 2011, 54, 7397.

(9) Wang, F.; Huang, K.; Yang, L.; Gong, J.; Tao, Q.; Li, H.; Zhao, Y.; Zeng, S.; Wua, X.; Stockigt, J.; Li, X.; Qu, J. Bioorg. Med. Chem. 2009, 17, 6380.

(10) Gažák, R.; Svobodová, A.; Psotová, J.; Sedmera, P.; Přikrylová, V.; Walterová, D.; Křen, V. Bioorg. Med. Chem. 2004, 12, 5677.

(11) Maitrejean, M.; Comte, G.; Barron, D.; El Kirat, K.; Conseil, G.; Di Pietro, A. Bioorg. Med. Chem. Lett. 2000, 10, 157.

(12) Křen, V.; Kubisch, J.; Sedmera, P.; Halada, P.; Přikrylová, V.; Jegorov, A.; Cvak, L.; Gebhardt, R.; Ulrichová, J.; Šimánek, V. J. Chem. Soc., Perkin Trans. 1 1997, 2467.

(13) Zarrelli, A.; Sgambato, A.; Petito, V.; De Napoli, L.; Previtera, L.; Di Fabio, G. Bioorg. Med. Chem. Lett. 2011, $21,4389$.

(14) De Napoli, L.; Di Fabio, G.; D'Onofrio, J.; Montesarchio, D. Chem. Commun. 2005, 2586; and references cited therein.

(15) Ettmayer, P.; Amidon, G. L.; Clement, B.; Testa, B. J. Med. Chem. 2004, 47, 2393.

(16) Natarajan, V.; Jeong, K. S.; Byeang, K. Curr. Med. Chem. 2003, 10, 1973.

(17) General Procedure for the Preparation of Phosphoramidite 3

To 3,5,7,20-tetra- $O$-acetylsilybin (2, $240.0 \mathrm{mg}, 0.37 \mathrm{mmol})$ dissolved in anhydrous $\mathrm{CH}_{2} \mathrm{Cl}_{2}(5 \mathrm{~mL})$, DIEA $(390 \mu \mathrm{L}$, $2.22 \mathrm{mmol}$ ), and 2-cyanoethyl- $N, N$-diisopropylaminochlorophosphoramidite $(107 \mu \mathrm{L}, 0.48 \mathrm{mmol})$ were mixed under argon. After 30 min the solution was diluted with EtOAc, and the organic phase was washed twice with brine and then concentrated. Silica gel chromatography of the residue (eluent $n$-hexane-EtOAc $=3: 7, \mathrm{v} / \mathrm{v}$, in the presence of $1 \%$ of $\left.\mathrm{Et}_{3} \mathrm{~N}\right)$, afforded desired compound $3(205.0 \mathrm{mg}$, $0.24 \mathrm{mmol})$ in a $65 \%$ yield. $R_{f}=0.8(n$-hexane-EtOAc $=3: 7$, $\mathrm{v} / \mathrm{v})$.

${ }^{1} \mathrm{H}$ NMR $\left(500 \mathrm{MHz}, \mathrm{CDCl}_{3}\right.$, r.t., mixture of diastereomers): $\delta=7.11-6.90(6 \mathrm{H}$, overlapped signals, H-13, H-15, H-16, H-18, H-21, H-22), 6.81 (1 H, s, H-8), 6.58 (1 H, s, H-6), $5.66(1 \mathrm{H}, \mathrm{d}, J=11.6 \mathrm{~Hz}, \mathrm{H}-3), 5.37(1 \mathrm{H}, \mathrm{d}, J=11.6 \mathrm{~Hz}, \mathrm{H}-$ 2), $5.03(1 \mathrm{H}, \mathrm{d}, J=7.6 \mathrm{~Hz}, \mathrm{H}-11), 4.13(1 \mathrm{H}, \mathrm{m}, \mathrm{H}-10)$, 3.90-3.60 $\left\{9 \mathrm{H}\right.$, complex signals, $\mathrm{OCH}_{3}, \mathrm{H}-23$,

$\left.\mathrm{OCH}_{2} \mathrm{CH}_{2} \mathrm{CN}, \mathrm{N}\left[\mathrm{CH}\left(\mathrm{CH}_{3}\right)_{2}\right]_{2}\right\}, 2.65(2 \mathrm{H}$, complex signals, $\left.\mathrm{OCH}_{2} \mathrm{CH}_{2} \mathrm{CN}\right), 2.37,2.32,2.29,2.04\left(12 \mathrm{H}\right.$, s for each, $\mathrm{CH}_{3}$ of acetyl groups), 1.25-1.15 $\{12 \mathrm{H}$, complex signals, $\left.\mathrm{N}\left[\mathrm{CH}\left(\mathrm{CH}_{3}\right)_{2}\right]_{2}\right\}$ ppm. ${ }^{13} \mathrm{C} \mathrm{NMR}\left(125 \mathrm{MHz}, \mathrm{CDCl}_{3}\right.$, r.t., mixture of diastereomers): $\delta=185.3(\mathrm{C}-4), 169.1$ (C-7), $168.7,167.8$ (CO of acetyl groups), 162.5 (C-5), 156.3 (C8a), 151.4 (C-19), 144.3 (C-20), 143.6 (C-16a), 140.2 (C12a), 134.8 (C-14), 123.0 (C-17), 120.8 (C-15), 119.9 (C22), 119.8, 117.6, $117.2\left(\mathrm{C}-13, \mathrm{C}-16, \mathrm{OCH}_{2} \mathrm{CH}_{2} \mathrm{CN}\right), 116.2$ (C-21), 111.4, 111.2, 110.6, 108.9 (C-6, C-8, C-18, C-4a), 81.0 (C-2), 76.2 (C-10), 75.8 (C-11), 73.3, 73.1 (C-3, C-3'),
62.6, $62.2\left(\mathrm{C}-23, \mathrm{C}-23^{\prime}\right), 58.8,58.5\left(\mathrm{OCH}_{2} \mathrm{CH}_{2} \mathrm{CN}\right), 56.0$ $\left(\mathrm{OCH}_{3}\right), 43.3,43.1\left\{\mathrm{~N}\left[\mathrm{CH}\left(\mathrm{CH}_{3}\right)_{2}\right]_{2}\right\}, 24.5\left\{\mathrm{~N}\left[\mathrm{CH}\left(\mathrm{CH}_{3}\right)_{2}\right]_{2}\right\}$, 21.1, 20.9, 20.7, $20.4\left(\mathrm{CH}_{3}\right.$ of acetyl groups $), 19.9$ $\left(\mathrm{OCH}_{2} \mathrm{CH}_{2} \mathrm{CN}\right) \mathrm{ppm} .{ }^{31} \mathrm{P} \mathrm{NMR}\left(161.98 \mathrm{MHz}, \mathrm{CDCl}_{3}\right): \delta=$ $152.9,150.2 \mathrm{ppm}$. ESI-HRMS (positive ions): $\mathrm{m} / \mathrm{z}$ calcd for $\mathrm{C}_{42} \mathrm{H}_{47} \mathrm{~N}_{2} \mathrm{O}_{15} \mathrm{P}: 850.2714$; found: $[\mathrm{MH}]^{+}:$851.2788; [MNa ${ }^{+}$ $=873.2606$.

(18) Janout, V.; Di Giorgio, C.; Regen, S. L. J. Am. Chem. Soc. 2000, 122, 2671.

(19) Wallimann, P.; Marti, T.; Fürer, A.; Diederich, F. Chem. Rev. 1997, 97, 1567.

(20) Swaan, P. W.; Hillgren, K. M.; Szoka, F. C. Jr.; Øie, S. Bioconjugate Chem. 1997, 8, 520.

(21) Banerjee, S. S.; Aher, N.; Patil, R.; Khandare, J. J. Drug Deliv. 2012, 2012, 103973.

(22) Knop, K.; Hoogenboom, R.; Fischer, D.; Schubert, U. S. Angew. Chem. Int. Ed. 2010, 49, 6288.

(23) Richter, Y.; Fischer, B. J. Biol. Inorg. Chem. 2006, 11, 1063.

(24) Kachur, A. V.; Manevich, Y.; Biaglow, J. E. Free Radical Res. 1997, 26, 399.

(25) Wolf, S.; Zismann, T.; Lunau, N.; Meier, C. Chem.-Eur. J. 2009, 15, 7656; and references cited therein.

(26) Maier, M. A.; Yannopoulos, C. G.; Mohamed, N.; Roland, A.; Fritz, H.; Mohan, V.; Just, G.; Manoharan, M. Bioconjugate Chem. 2003, 14, 18.

(27) General Procedure for the Synthesis of Conjugates 5 (AE)

Derivative 3 ( $150 \mathrm{mg}, 0.22 \mathrm{mmol}$ ) and the requisite compound $\mathbf{A}-\mathbf{E}(0.21 \mathrm{mmol}$, previously dried and kept under reduced pressure, were reacted with a $0.45 \mathrm{M}$ tetrazole solution in anhydrous $\mathrm{MeCN}(1.0 \mathrm{~mL}, 0.45 \mathrm{mmol})$. The reaction was left under stirring at r.t. and monitored by TLC with an eluent system $n$-hexane-EtOAc $=1: 1(\mathrm{v} / \mathrm{v})$. After 1 $\mathrm{h}$, a $5.5 \mathrm{M} t$-BuOOH solution in decane $(100 \mu \mathrm{L})$ was added to the mixture and left stirring at r.t. After $30 \mathrm{~min}$ the reaction mixture was diluted with $\mathrm{CHCl}_{3}$, transferred into a separatory funnel, washed three times with $\mathrm{H}_{2} \mathrm{O}$, concentrated under reduced pressure, and purified by flash chromatography, eluting with $n$-hexane-EtOAc $=7: 3(\mathrm{v} / \mathrm{v})$, to afford pure $\mathbf{4 a - e}$ as a yellow-brown amorphous powder. Treatment with concd aq $\mathrm{NH}_{3}$ in $\mathrm{MeOH}(1: 1, \mathrm{v} / \mathrm{v})$ for $1 \mathrm{~h}$ at r.t., led to full removal of the acetyl and 2-cyanoethyl groups. The concentrated mixture was then purified on a silica gel column, eluting with $\mathrm{CHCl}_{3}-\mathrm{MeOH}$ containing increasing proportions of $\mathrm{MeOH}$. Compounds $\mathbf{5 a}-\mathbf{e}$ thus obtained were converted into the corresponding sodium salts by cation exchange on a DOWEX $\left(\mathrm{Na}^{+}\right.$form) resin to obtain homogeneous samples in good yield $(55-72 \%)$. See the Supporting Information.

Compound 5a: NMR spectra of this compound showed dramatic line broadening, diagnostic of a slow equilibrium on the NMR time scale, which could suggest a strong propensity toward aggregation in $\mathrm{CDCl}_{3} .{ }^{1} \mathrm{H}$ NMR (500 $\mathrm{MHz}, \mathrm{CDCl}_{3}$, r.t., mixture of diastereomers): $\delta=7.02-6.60$ $(6 \mathrm{H}$, complex signals), $5.85(2 \mathrm{H}$, br s $), 5.59(1 \mathrm{H}, \mathrm{dd}, J=$ $11.9,11.7 \mathrm{~Hz}), 5.11(2 \mathrm{H}$, complex signals), $4.78(1 \mathrm{H}, \mathrm{br} \mathrm{s})$, 4.04 ( $1 \mathrm{H}$, br signal), 3.95-3.63 (6 H, complex signals), 2.05 ( $2 \mathrm{H}$, br signal), 1.92-0.99 (26 H, complex signals), 0.85 (3 $\mathrm{H}, \mathrm{br} \mathrm{s}), 0.79(3 \mathrm{H}, \mathrm{d}, J=4.8 \mathrm{~Hz}), 0.74(6 \mathrm{H}, \mathrm{br} \mathrm{s}), 0.55(3 \mathrm{H}$, s) ppm. ${ }^{31} \mathrm{P} \mathrm{NMR}\left(161.98 \mathrm{MHz}, \mathrm{CDCl}_{3}\right): \delta=-0.83 \mathrm{ppm}$. HRMS (MALDI-TOF, negative ions): $\mathrm{m} / \mathrm{z}$ calcd for $\mathrm{C}_{52} \mathrm{H}_{66} \mathrm{O}_{13} \mathrm{P}$ : 929.4246; found: $929.4246[\mathrm{M}-\mathrm{H}]^{-}$. Compound 5b: ${ }^{1} \mathrm{H}$ NMR $\left(500 \mathrm{MHz}, \mathrm{CD}_{3} \mathrm{OD}\right.$, r.t., mixture of diastereomers): $\delta=7.11-6.72$ ( $6 \mathrm{H}$, complex signals), 5.91 $(1 \mathrm{H}, \mathrm{d}, J=1.5 \mathrm{~Hz}), 5.85(1 \mathrm{H}, \mathrm{m}), 4.98-4.75(2 \mathrm{H}$, complex signal), $4.41(1 \mathrm{H}, \mathrm{d}, J=11.5 \mathrm{~Hz}), 4.21-4.05(5 \mathrm{H}, \mathrm{m}), 3.88$ 3.68 (24 H, complex signals) ppm. ${ }^{31} \mathrm{P}$ NMR (161.98 MHz, 
$\mathrm{CDCl}_{3}$ ): $\delta=3.1 \mathrm{ppm}$. HRMS (MALDI-TOF, negative ions): $\mathrm{m} / \mathrm{z}$ calcd for $\mathrm{C}_{36} \mathrm{H}_{44} \mathrm{O}_{18} \mathrm{P}: 795.2271$; found: $795.2271[\mathrm{M}-$ $\mathrm{H}]^{-}$.

Compound 5c: ${ }^{1} \mathrm{H}$ NMR $\left(500 \mathrm{MHz}, \mathrm{CD}_{3} \mathrm{OD}\right.$, r.t., mixture of diastereomers): $\delta=7.79(1 \mathrm{H}, \mathrm{s}), 7.11-6.72(6 \mathrm{H}$, complex signals), $6.41(1 \mathrm{H}, \mathrm{dd}, J=6.8,6.8 \mathrm{~Hz}), 5.91(1 \mathrm{H}, \mathrm{d}, J=1.5$ $\mathrm{Hz}), 5.85(1 \mathrm{H}, \mathrm{m}), 4.97-4.74(2 \mathrm{H}$, complex signal), 4.65 (1 $\mathrm{H}, \mathrm{m}), 4.41(1 \mathrm{H}, \mathrm{d}, J=11.5 \mathrm{~Hz}), 4.22-4.10(4 \mathrm{H}, \mathrm{m}), 4.00$ $3.85(5 \mathrm{H}$, complex signals), $2.44(2 \mathrm{H}, \mathrm{m}), 1.99(3 \mathrm{H}, \mathrm{s})$ ppm. ${ }^{31} \mathrm{P}$ NMR $\left(161.98 \mathrm{MHz}, \mathrm{CDCl}_{3}\right): \delta=3.3 \mathrm{ppm}$. HRMS (MALDI-TOF, negative ions): $\mathrm{m} / \mathrm{z}$ calcd for $\mathrm{C}_{35} \mathrm{H}_{34} \mathrm{~N}_{2} \mathrm{O}_{17} \mathrm{P}$ : 785.1600; found: $785.1602[\mathrm{M}-\mathrm{H}]^{-}$.

Compound 5d: ${ }^{1} \mathrm{H}$ NMR $\left(500 \mathrm{MHz}, \mathrm{CD}_{3} \mathrm{OD}\right.$, r.t., mixture of diastereomers): $\delta=8.56(1 \mathrm{H}, \mathrm{s}), 8.29(1 \mathrm{H}, \mathrm{s}), 7.11-6.72(6$ $\mathrm{H}$, complex signals), $6.05(1 \mathrm{H}, \mathrm{d}, J=6.0 \mathrm{~Hz}), 5.91(1 \mathrm{H}, \mathrm{d}$, $J=1.5 \mathrm{~Hz}), 5.85(1 \mathrm{H}, \mathrm{m}), 4.90(2 \mathrm{H}$, complex signals $), 4.63$
$(1 \mathrm{H}, \mathrm{m}), 4.54(1 \mathrm{H}, \mathrm{m}), 4.41(1 \mathrm{H}, \mathrm{d}, J=11.5 \mathrm{~Hz}), 4.35(1$ $\mathrm{H}, \mathrm{m}), 4.20(2 \mathrm{H}, \mathrm{m}), 4.21-4.05(3 \mathrm{H}, \mathrm{m}), 3.88-3.68(3 \mathrm{H}, \mathrm{s})$ ppm. ${ }^{31} \mathrm{P}$ NMR $\left(161.98 \mathrm{MHz}, \mathrm{CDCl}_{3}\right): \delta=3.1 \mathrm{ppm}$. HRMS (MALDI-TOF, negative ions): $m / z$ calcd for $\mathrm{C}_{35} \mathrm{H}_{33} \mathrm{~N}_{5} \mathrm{O}_{16} \mathrm{P}$ : 810.1665; found: $810.1666[\mathrm{M}-\mathrm{H}]^{-}$.

Compound 5e: ${ }^{1} \mathrm{H}$ NMR $\left(500 \mathrm{MHz}, \mathrm{CD}_{3} \mathrm{OD}\right.$, r.t., mixture of diastereomers): $\delta=7.08-6.81$ (6 H, complex signals), 5.95$5.89(2 \mathrm{H}, \mathrm{m}), 5.07(1 \mathrm{H}, \mathrm{m}), 4.97(1 \mathrm{H}, \mathrm{m}), 4.52(2 \mathrm{H}, \mathrm{m})$, $4.24(1 \mathrm{H}, \mathrm{m}), 4.06(1 \mathrm{H}, \mathrm{m}), 3.95-3.68(7 \mathrm{H}$, complex signals), $3.30(1 \mathrm{H}, \mathrm{m}), 2.01-0.70(33 \mathrm{H}$, complex signals $)$ ppm. ${ }^{31} \mathrm{P}$ NMR ( $\left.161.98 \mathrm{MHz}, \mathrm{CDCl}_{3}\right): \delta=4.3 \mathrm{ppm}$. ESI-MS (positive ions): $m / z$ calcd for $\mathrm{C}_{49} \mathrm{H}_{63} \mathrm{O}_{16} \mathrm{P}: 938.39$; found: $939.48[\mathrm{MH}]^{+} ;[\mathrm{MNa}]^{+}=961.37$. HRMS (MALDI-TOF, negative ions): $m / z$ calcd for $\mathrm{C}_{49} \mathrm{H}_{62} \mathrm{O}_{16} \mathrm{P}: 937.3781$; found: $937.3782[\mathrm{M}-\mathrm{H}]^{-}$.

(28) Stawinski, J.; Kraszewski, A. Acc. Chem Res. 2002, 35, 952. 